\title{
Homocysteine Slovakia study: study design and occurrence of hyperhomocysteinaemia and other risk factors
}

\author{
Lietava $\mathbf{J}^{1}$, Vohnout $\mathrm{B}^{1,3}$, Dukat $\mathrm{A}^{1}$, Fodor $\mathrm{GJ}^{2}$ \\ 2nd Department of Internal Medicine, Comenius University, Bratislava, Slovakia. jan.lietava@yahoo.com
}

\begin{abstract}
Background: Slovakia belong to the European Union countries with the high incidence and prevalence of cardiovascular diseases in general and IHD in particular.

Method: Homocystein-Slovakia is crossectional population study realised in subjects in high risk age 35-75 years in two groups. The first consists of patients with verified stable ischemic heart disease (IHD) $(M=152 ; F=167)$ aged $60.8 \pm 9.37$ yrs (males) res. $63.1 \pm 7.56$ years (females) (NS) who were randomly selected from two cardiological registrars. Second population was formed by general population who were dichotomised according their medical records into IHD patients $(M=31, F=53)$ and apparently healthy controls $(M=47 ; F=55)$, the later in significantly younger age as patients, but in same age for intergender comparison $49.6 \pm 10.3$ vs $46.6 \pm 9.2$ yrs (NS). Results: We found very high prevalence of classic as well as newer risk factors and risk markers both in IHD patients and in controls.

Increased homocysteinen (Hcy $>15 \mu \mathrm{mo} / \mathrm{l}$ for males and Hcy $>13 \mu \mathrm{mo} / \mathrm{l}$ for females) was found even in $32.9 \%$ of patients and $13.6 \%$ of controls $(p<0.001)$. Comparison of regulating vitamins levels between IHD patients and controls demonstrated similar prevalence.

Conclusion: Homocystein Slovakia study found very high prevalence of hyperhomocysteinemia in patients with stable ischemic heart disease. Even the prevalence in healthy controls correspond to data resported for MI patients in Western countries. Vitamins regulating metabolism of homocysteine also shown high prevalence, however, without differences between IHD pacients and controls (Tab. 5, Fig. 1, Ref. 27). Full Text in PDF www.elis.sk.

Key words: homocystein Slovakia, epidemiology, risk factors, vitamins B6, B12 and folic acid.
\end{abstract}

Increased plasmatic level of homocysteine (HCy) is associated with serious clinical manifestation of atherosclerosis such as myocardial infarction (MI), stroke or peripheral vascular disease $(1,2)$.

Population studies have documented a prevalence of hyperhomocysteinaemia at about $10 \%$, however, in a selected group of patients with apparent ischaemic heart disease (IHD) the prevalence reached up to $30-40 \%(3,4)$. Nested case-control studies showed that a $5-\mu \mathrm{mol} / \mathrm{L}$ increment in HCy results in a $20-30 \%$ increase in cardiovascular risk (5), however despite the decrease in homocysteine, intervention did not decrease the cardiovascular or total mortality $(6,7)$.

Homocysteine is formed in the body from methionin. Methionin is metabolised via re-methylation and transsulphuration. These processes depend on vitamins B12, B6 and folic acid, thus regulated through nutrition.

\footnotetext{
${ }^{12}$ nd Department of Internal Medicine, Comenius University, Bratislava, Slovakia, ${ }^{2}$ University of Ottawa Heart Institute, Ottawa Canada, and ${ }^{3} \mathrm{De}-$ partment of Physiological and Clinical Nutrition and National Reference Centre for Familial Hyperlipoproteinaemias, Slovak Medical University, Bratislava, Slovakia
}

Address for correspondence: J. Lietava, MD, PhD, 2nd Department of Internal Medicine, Comenius University, Mickiewiczova 13, SK-813 69 Bratislava, Slovakia.

Acknowledgements: Investigators would like to express their gratitude to all sponsors, co-workers and supporters of our project.
Slovakia has a very high incidence and prevalence of cardiovascular diseases in general, and IHD in particular (8), so far however the prevalence of homocysteine and related vitamins has not been studied sufficiently.

The goal of the present study was to investigate the prevalence of hyperhomocysteinaemia and its association with risk factors of atherosclerosis in patients with ischaemic heart disease (IHD) and in the general population from selected rural and urban districts of Slovakia.

\section{Materials and methods}

We randomly selected both, male and female Caucasian patients between 35-75 years of age with known IHD from a mixed urban/rural district of Nové Zámky (standardised mortality: (SM) $=10.4$ ), from the city of Bratislava (the capital of the Slovak Republic) $(\mathrm{SM}=9.8)$ and we invited all residents from the village of Vel'ký Lom and its surrounding area (district Vel'ký Krtíš) (SM =12.6) (9). The age range was chosen to estimate HCy levels in age groups with expected high risk of cardiovascular events. Patients suffering from megaloblastic anaemia, malabsorption or inflammatory bowel disease, serum-positive arthritis, drug addicts, toxicomania and alcoholism (Jelinek IV), chronic renal disease of stages III and IV according to K/DOQI, extensive trauma less than three months before the study, maniodepressive psychosis, 
Tab. 1. Selection process of Homocystein Slovakia participants.

\begin{tabular}{lclc}
\hline Nové Zámky, Bratislava & \multicolumn{3}{c}{ Vel'ký Lom } \\
\hline Registars & 6228 & Population & 325 \\
Age (35-75 years) & 3627 & Age (35-75 years) & 223 \\
Proved IHD & 1281 & & \\
Randomised & 700 & & \\
Invited & 300 & Examined & 186 \\
Examined & 247 & Proved IHD & 84 \\
Participation & $82.3 \%$ & Participation & $83.4 \%$ \\
\hline
\end{tabular}

Abbreviations: IHD - ischemic heart disease

gastric surgery, immobility, acute MI or coronary intervention less than three months before study, on cancer chemotherapy or radiotherapy less than three months before study, on vegan diet, and polyvitamin substitution were excluded from the study. To obtain a high participation rate we published a series of articles in local newspapers explaining the goals of the study, and the patients were invited by telephone to participate. Local authorities supported the study by explaining its goals through local radio networks and by providing facilities to conduct the investigation.

IHD patients were selected from the register of two district cardiologists in Nové Zámky and one in Bratislava. IHD was defined as 1) MI requiring hospitalisation 2) and/or typical angina pectoris (AP) or 3) proven and treated silent IHD documented by medical report and ECG signs of ischaemia.

Two trained research assistants reviewed all medical records from the registers and included or excluded all subjects according to the set criteria. Subsequently the principal investigator examined all records and confirmed inclusion and exclusion criteria.

All adult residents from Vel'ký Lom and its surrounding area in age range of 35-75 years were invited to participate in the study. Their medical records were assessed in the offices of family physicians and the patients were excluded based on the principles described above. The results of the selection process according to the inclusion and exclusion criteria in individual districts are shown in Table 1.

At the end of the screening process, 130 patients in Nové Zámky, 117 in Bratislava, and 186 subjects in Vel'ký Lom were included in the study (Tab.1).

The selected patients and respondents had a brief medical examination and were interviewed by a physician using a standardised questionnaire. All physicians were pre-trained for filling in the standardised questionnaire. The questionnaire recorded personal and family history, nutritional habits assessing the basic pattern of meat, vegetable, fruit and salt intake, smoking status (current, former smoker, never smoked), physical activity, and risk factor evaluation. Socioeconomic status was evaluated by income and level of education (primary school, secondary school, university). Professional status was evaluated according to ILO standard classification of occupations (11). IHD part of the questionnaire was based on the Rose Protocol for Angina Pectoris (12). Blood pressure was measured in a separate quiet room after a five-minute rest. Three standard mercury sphygmomanometer measurements were taken from the right arm in a sitting position. Only the last two measurements were considered in the study.
We computed the absolute CHD risk according to recommendations of European Heart Society (13) using a special software developed for the study. Smoking index of risk assessment in current and former smokers was calculated (14).

Venous blood samples were collected into EDTA-Potasium tubes to obtain plasma and into standard tubes to obtain serum after overnight fasting without cubital compression. Blood for plasma was immediately centrifuged in a cooled centrifuge $\left(4^{\circ} \mathrm{C}\right)$ for 30 minutes; blood for serum was allowed to clot for 15 minutes at room temperature in range of $22-25^{\circ} \mathrm{C}$ and underwent the same centrifugation procedure. Supernatants and sediment from plasma blood samples were removed and stored in cryovials. All three blood derivates were stored immediately after the procedure at $-70{ }^{\circ} \mathrm{C}$. Plasma levels of total cholesterol (TC) and triglycerides (TG) were measured enzymatically; ApoB and apoAI levels were measured by immunoturbidimetric method; HDL cholesterol (HDL-C) was determined directly by commercial kit (Genzyme) on autoanalyser (Hitachi 911, Roche, Switzerland). LDL-C was both, evaluated by direct method for LDL-C measurement (Randox, UK) and calculated using the Friedewald formula only if the triglyceride concentration was below $4.5 \mathrm{mmol} / \mathrm{l}$ (15). Glycaemia, $\mathrm{Na}, \mathrm{K}, \mathrm{Ca}^{2+}, \mathrm{Fe}, \mathrm{Cl}, \mathrm{Mg}$, creatinin, BUN, albumin, bilirubin, ALT, AST, and GMT were measured using standard methods. EDTA vacutainers for homocysteine measurement were kept on ice and centrifuged within 15 minutes after blood drawing in cooled centrifuge $\left(3000 \mathrm{rpm} / 30 \mathrm{~min} / 4{ }^{\circ} \mathrm{C}\right)$. The plasma was then stored at $-80{ }^{\circ} \mathrm{C}$ until analysed by high-performance liquid chromatography (HPLC) using a standard kit (CHROMSYSTEMS Instruments \& Chemicals $\mathrm{GmbH}$, Munich). The interassay coefficient of variation of the method was $4.6 \%$. Moreover, in a subset of 100 samples, plasma Hcy levels were analysed using also ELISA method. Vitamin $\mathrm{B}_{6}$ levels in serum were measured using HPLC method (CHROMSYSTEMS Instruments \& Chemicals GmbH, Munich, Germany), serum vitamin B12 and folic acid levels were analysed by ELISA (Roche Diagnostics Corp., USA). The blood cells count and urinalysis were determined by standard methods. Twenty-four-hour urine was collected, actual volume, density and temperature were measured, and urine samples were stored at $-80^{\circ} \mathrm{C}$.

Uric acid was measured with spectrophotometer at $456 \mathrm{~nm}$ (Hitachi 911, Roche, Switzerland), plasmatic total antioxidant status (TAS) was stated with colorimeter using original kit for TAS (Randox, Cat no NX 2332, Randox Laboratories, Cromlech, $\mathrm{UK})$. Blood for total glutathione (tGSH) and oxidised glutathione (GSSG) measurement was deproteinised by $10 \%$ sulphosalicylic acid (SSA) (400 $\mu 110 \%$ SSA, $750 \mu$ l blood), centrifuged at 4 ${ }^{\circ} \mathrm{C}$ at $10,000 \mathrm{rpm}$ for 12 minutes and the supernatant was stored in cryovials at $-70{ }^{\circ} \mathrm{C}$ until spectrophotometrical analysis at 410 $\mathrm{nm}$ using microplate reader (TECAN Spectra Fluor, Austria) in kinetic-type of reaction (16). The Schiff base substance was measured with fluorometer using excitation wave length of $335 \mathrm{~nm}$ and emission wave length of $430 \mathrm{~nm}$ after repeated extraction in ethanolether mixture. $\operatorname{IgG}, \operatorname{IgM}$ and $\operatorname{IgE}$ antibody to Clamydia trachomatis, C.pneumoniae and C.psittaci were measured from serum using ELISA and microimmunofluorescence test (Lab- 
Tab. 2. Antropometric and laboratory characteristics of males.

\begin{tabular}{|c|c|c|c|c|c|c|}
\hline \multicolumn{7}{|c|}{ Males } \\
\hline \multirow[t]{2}{*}{ Parameter } & \multicolumn{4}{|c|}{ IHD patients } & \multicolumn{2}{|c|}{ Controls } \\
\hline & Nové Zámky (N=60) & Bratislava $(\mathrm{N}=64)$ & Vel'ký Lom (N=31) & Sign. & Vel'ký Lom (N=47) & Sign. \\
\hline Age [yrs] & $61.2 \pm 8.2$ & $61.0 \pm 10.6$ & $58.7 \pm 9.5$ & NS & $49.6 \pm 10.3$ & 0.001 \\
\hline BMI [kg/m2] & $29.2 \pm 3.9$ & $28.7 \pm 3.3$ & $29.1 \pm 5.3$ & NS & $100.0 \pm 11.3$ & 0.06 \\
\hline Waist $[\mathrm{cm}]$ & $105,9 \pm 9.8$ & $102.2 \pm 8.8$ & $106.0 \pm 11.7$ & NS & $0.95 \pm 0.06$ & 0.01 \\
\hline $\mathrm{SBP}[\mathrm{mmHg}]$ & $146.2 \pm 21.7$ & $138.3 \pm 24.2$ & $150.1 \pm 27.3$ & 0.051 & $143.6 \pm 20.8$ & NS \\
\hline $\mathrm{DBP}[\mathrm{mmHg}]$ & $88.0 \pm 11.5$ & $86.1 \pm 12.3$ & $95.9 \pm 12.3$ & 0.003 & $93.4 \pm 13.5$ & 0.05 \\
\hline $\mathrm{TCH}[\mathrm{mmol} / \mathrm{l}]$ & $5.6 \pm 1.1$ & $5.4 \pm 0.9$ & $5.7 \pm 1.1$ & NS & $6.2 \pm 1.1$ & 0.001 \\
\hline LDL-C $[\mathrm{mmol} / 1]$ & $3.7 \pm 1.0$ & $3.4 \pm 0.8$ & $3.6 \pm 0.9$ & NS & $4.1 \pm 1.0$ & 0.002 \\
\hline $\mathrm{HDL}-\mathrm{C}[\mathrm{mmol} / \mathrm{l}]$ & $1.2 \pm 0.3$ & $1.2 \pm 0.3$ & $1.3 \pm 0.5$ & NS & $1.4 \pm 0.3$ & 0.001 \\
\hline $\mathrm{TG}[\mathrm{mmol} / \mathrm{l}]$ & $1.9 \pm 1.5$ & $1.8 \pm 1.0$ & $1.6 \pm 0.9$ & NS & $1.6 \pm 0.8$ & 0.06 \\
\hline Apo-B $[\mathrm{g} / 1]$ & $1.1 \pm 0.3$ & $1.0 \pm 0.2$ & $1.1 \pm 0.2$ & NS & $1.1 \pm 0.2$ & 0.02 \\
\hline Apo-AI [g/l] & $1.0 \pm 0.1$ & $1.0 \pm 0.1$ & $1.1 \pm 0.2$ & 0.001 & $1.2 \pm 0.2$ & 0.01 \\
\hline AI & $5.1 \pm 1.3$ & $4.9 \pm 1.2$ & $4.6 \pm 1.2$ & NS & $4.6 \pm 1.2$ & NS \\
\hline Glycemia [mmol/1] & $6.7 \pm 2.2$ & $6.9 \pm 2.6$ & $6.8 \pm 2.6$ & NS & $6.1 \pm 2.4$ & NS \\
\hline $\mathrm{Hb}[\mathrm{g} / \mathrm{dl}]$ & $145 \pm 12.0$ & $144 \pm 10.9$ & $147 \pm 16.3$ & NS & $154 \pm 14.3$ & 0,001 \\
\hline Leu $[* 109 / 1]$ & $6.6 \pm 1.6$ & $6.8 \pm 3.2$ & $6.9 \pm 1.9$ & NS & $6.5 \pm 1.3$ & NS \\
\hline Plt $[* 109 / 1]$ & $189.7 \pm 53.6$ & $191.4 \pm 59.1$ & $222.1 \pm 79.7$ & NS & $206.7 \pm 45.4$ & NS \\
\hline $\mathrm{Fe}[\mathrm{mmol}[1]$ & $19.0 \pm 6.1$ & $18.3 \pm 6.0$ & $17.6 \pm 8.8$ & NS & $17.9 \pm 6.4$ & NS \\
\hline $\mathrm{Bi}[\mathrm{mmol}[1]$ & $12.5 \pm 7.9$ & $10.3 \pm 5.2$ & $12.1 \pm 6.0$ & NS & $11.6 \pm 6.3$ & NS \\
\hline GMT $[\mu \mathrm{mo} / 1]$ & $0.70 \pm 0.49$ & $0.56 \pm 0.37$ & $0.65 \pm 0.57$ & MS & $0.64 \pm 0.56$ & NS \\
\hline ALT $[\mu \mathrm{kat} / 1]$ & $0.45 \pm 0.24$ & $0.44 \pm 0.25$ & $0.44 \pm 0.38$ & NS & $0.59 \pm 0.41$ & NS \\
\hline $\operatorname{ALP}[\mu \mathrm{mo} / 1]$ & $3.3 \pm 0.8$ & $3.1 \pm 0.8$ & $3.4 \pm 1.1$ & NS & $3.2 \pm 0.7$ & NS \\
\hline Creatinine $[\mu \mathrm{mo} / 1]$ & $102 \pm 15$ & $107 \pm 20$ & $92 \pm 12$ & 0.001 & $90 \pm 11$ & 0.001 \\
\hline $\mathrm{UA}[\mu \mathrm{mo} / 1]$ & $393 \pm 83$ & $389 \pm 94$ & $358 \pm 104$ & $\mathrm{NS}-0.07$ & $360 \pm 88$ & NS \\
\hline Hcy $[\mu \mathrm{mo} / 1]$ & $14.4 \pm 7.4$ & $14.3 \pm 5.0$ & $13.8 \pm 5.3$ & $\mathrm{NS}-0.06$ & $12.3 \pm 4.9$ & 0.03 \\
\hline Vit. B6 [nmol/1] & $0.80 \pm 0.33$ & $1.16 \pm 0.52$ & $1.06 \pm 0.51$ & 0.001 & $1.11 \pm 0.58$ & NS \\
\hline Vit. B12 [pmol/1] & $276 \pm 104.1$ & $282 \pm 117.9$ & $249 \pm 75.3$ & NS & $298 \pm 115.8$ & NS \\
\hline Folic acid [nmol/1] & $13.7 \pm 6.7$ & $14.3 \pm 9.3$ & $10.2 \pm 6.7$ & 0.002 & $10.8 \pm 4.8$ & NS \\
\hline hsCRP $[\mathrm{mg} / \mathrm{dl}]$ & $3.0 \pm 2.8$ & $1.8 \pm 1.9$ & $2.6 \pm 2.9$ & $\mathrm{NS}-0.06$ & $2.0 \pm 2.1$ & NS \\
\hline
\end{tabular}

BMI - body mass index, SBP - systolic blood pressure, DBP - diastolic blood pressure, TCH - total cholesterol, LDL - LDL cholesterol, HDL - HDL cholesterol, TG triglycerides, ApoB- Apolipoprotein B, ApoA1 - Apolipoprotein A1, AI- atherogenic index, Hb - haemoglobin, Leu - leukocytes, Plt - platelets, Fe - iron, Bi - bilirubin, $\mathrm{UA}$ - uric acid, Hcy - homocystein, vit. B6 - vitamin B6, vit. B12 - vitamin B12, hsCRP - high sensitivity C-reactive protein, IHD - ischemic heart disease

systems, Finland). All the measurements done in the study were performed in certified laboratories.

Height, weight, waist and hip circumferences were measured according to standard protocols.

\section{Ethics}

The study was approved by the Ethics Committee of the Medical School of Comenius University in Bratislava, and all subjects signed the informed consent.

\section{Results}

Main characteristics of the groups by IHD status and geographical area are listed in Tables 2 and 3. There is a high occurrence of risk factors for atherosclerosis in all groups (Tabs 4 and 5).

In general, all groups were overweight, with majority of subjects with a BMI $>25 \mathrm{~kg} / \mathrm{m}^{2}$; a substantial proportion of the subjects exceeded the recommended levels of total and LDL cholesterol and HDL cholesterol (with exception of both female and male control subjects from Vel'ký Lom). Moreover, in more than $50 \%$ of IHD patients we detected increased systolic or diastolic blood pressure, namely over 140/90 mm Hg. Even in the control population, a high proportion of hypertensive subjects were revealed. There was a high- er prevalence of smokers in both IHD and control subjects from the rural area in Vel'ký Lom, compared to IHD patients in Nové Zámky and Bratislava. We have found a high level of classic risk factors and other risk factors, as well as absolute risk for cardiac events, namely $61 \%$ to $88 \%$ of IHD patients (depending on region and gender) had an absolute 10 years risk $\geq 20 \%$ compared to $15 \%$ and $26 \%$ of control females and males from Vel'ký Lom (Tabs 4 and 5).

When we compared personal history data obtained through the questionnaire filled in by patients with their medical records, we unexpectedly found a high number of IHD patients, both in Nové Zámky and Bratislava who were not aware of their diagnoses. In fact, $6.3 \%$ of patients in Nové Zámky and $4.8 \%$ in Bratislava were not aware of their diagnoses of AP, $6.3 \%$ and $3.2 \%$ of diagnoses of MI and $9.4 \%$ and $6.6 \%$ of diagnoses of hypertension. A similar situation was in males from Vel'ký Lom. These respondents considered themselves healthy even though they had been on disability benefit due to IHD or stroke (Fig. 1).

The mean plasma HCy level in men with IHD from Nové Zámky, Bratislava and Vel'ký Lom was $14.4 \pm 7.4,14.3 \pm 5.0$ and $13.8 \pm 5.3 \mu \mathrm{mol} / 1$, and $12.3 \pm 4.9 \mu \mathrm{mol} / \mathrm{l}$ in control men from Vel'ký Lom, respectively. In women with IHD, plasma Hcy levels were $12.6 \pm 6.4,11.0 \pm 3.0,12.2 \pm 5.3 \mu \mathrm{mol} / 1$ in Nové Zámky, Bratislava and Vel'ký Lom groups, respectively, compared to $8.6 \pm 3.2 \mu \mathrm{mol} / 1$ 
Tab. 3. Antropometric and laboratory characteristics of females.

\begin{tabular}{|c|c|c|c|c|c|c|}
\hline \multicolumn{7}{|c|}{ Females } \\
\hline \multirow[t]{2}{*}{ Parameter } & \multicolumn{4}{|c|}{ IHD patients } & \multicolumn{2}{|c|}{ Controls } \\
\hline & Nové Zámky $(\mathrm{N}=70)$ & Bratislava $(\mathrm{N}=53)$ & Vel'ký Lom $(\mathrm{N}=53)$ & Sign. & Vel'ký Lom $(\mathrm{N}=55)$ & Sign. \\
\hline Age [yrs] & $62.5 \pm 6.6$ & $66.1 \pm 7.6$ & $60.2 \pm 8.5$ & 0.001 & $46.6 \pm 9.2$ & 0.001 \\
\hline BMI $[\mathrm{kg} / \mathrm{m} 2]$ & $31.6 \pm 4.8$ & $28.8 \pm 5.1$ & $32.1 \pm 5.5$ & 0.002 & $28.8 \pm 6.6$ & 0.01 \\
\hline Waist [cm] & $98.3 \pm 11.0$ & $92.3 \pm 13.9$ & $102.2 \pm 13.1$ & 0.05 & $90.0 \pm 16.2$ & 0.001 \\
\hline $\mathrm{SBP}[\mathrm{mmHg}]$ & $158.6 \pm 20.5$ & $154.5 \pm 25.4$ & $157.1 \pm 23.4$ & NS & $138.7 \pm 29.4$ & 0.001 \\
\hline $\mathrm{DBP}[\mathrm{mmHg}]$ & $94.0 \pm 8.5$ & $91.2 \pm 11.4$ & $96.8 \pm 12.1$ & NS & $87.7 \pm 14.6$ & 0.005 \\
\hline TCH [mmol/l] & $6.0 \pm 1.0$ & $6.2 \pm 1.4$ & $6.6 \pm 1.1$ & 0.03 & $6.2 \pm 1.1$ & NS \\
\hline LDL-C [mmol/1] & $4.0 \pm 0.9$ & $4.2 \pm 1.2$ & $4.4 \pm 1.0$ & 0.03 & $4.1 \pm 1.0$ & NS \\
\hline $\mathrm{HDL}-\mathrm{C}[\mathrm{mmol} / \mathrm{l}]$ & $1.3 \pm 0.3$ & $1.4 \pm 0.3$ & $1.5 \pm 0.3$ & NS- 0.07 & $1.5 \pm 0.3$ & $\mathrm{NS}-0.06$ \\
\hline TG [mmol/1] & $1.8 \pm 0.9$ & $1.4 \pm 0.7$ & $1.6 \pm 0.8$ & NS & $1.4 \pm 0.6$ & NS \\
\hline Apo-B [g/l] & $1.0 \pm 0.2$ & $1.0 \pm 0.2$ & $1.1 \pm 0.2$ & NS & $1.0 \pm 0.2$ & NS \\
\hline Apo-AI [g/l] & $1.2 \pm 0.1$ & $1.1 \pm 0.2$ & $1.2 \pm 0.2$ & 0.001 & $1.2 \pm 0.1$ & NS \\
\hline $\mathrm{AI}$ & $4.9 \pm 1.1$ & $4.7 \pm 1.4$ & $4.7 \pm 1.1$ & NS & $4.4 \pm 1.2$ & NS \\
\hline Glycémia $[\mathrm{mmol} / \mathrm{l}]$ & $7.2 \pm 3.1$ & $6.1 \pm 1.6$ & $6.5 \pm 2.2$ & 0.05 & $5.8 \pm 1.3$ & 0.008 \\
\hline $\mathrm{Hb}[\mathrm{g} / \mathrm{dl}]$ & $140 \pm 10.2$ & $136 \pm 11.8$ & $139 \pm 12.6$ & NS & $138 \pm 10.0$ & NS \\
\hline Leu [*109/1] & $6.1 \pm 1.9$ & $6.3 \pm 1.8$ & $6.9 \pm 1.6$ & 0.01 & $7.3 \pm 1.6$ & 0.004 \\
\hline Plt [*109/1] & $255.5 \pm 54.4$ & $277.7 \pm 96.3$ & $244.4 \pm 66.7$ & NS & $264.4 \pm 60.0$ & $\mathrm{NS}-0.07$ \\
\hline $\mathrm{Bi}[\mathrm{mmol}[1]$ & $13.3 \pm 4.8$ & $12.6 \pm 3.8$ & $12.7 \pm 4.3$ & NS & $10.8 \pm 3.2$ & 0.03 \\
\hline GMT $[\mu \mathrm{mo} / 1]$ & $0.31 \pm 0.20$ & $0.33 \pm 0.30$ & ???? & NS & $0.31 \pm 0.24$ & NS \\
\hline ALT $[\mu \mathrm{kat} / 1]$ & $0.27 \pm 0.20$ & $0.26 \pm 0.12$ & $0.33 \pm 0.23$ & NS & $0.27 \pm 0.21$ & NS \\
\hline $\operatorname{ALP}[\mu \mathrm{mo} / 1]$ & $3.2 \pm 0.9$ & $3.2 \pm 0.9$ & $3.4 \pm 1.0$ & NS & $2.8 \pm 1.0$ & 0.003 \\
\hline Creatinine $[\mu \mathrm{mo} / \mathrm{l}]$ & $89 \pm 13$ & $90 \pm 15$ & $84 \pm 19$ & 0.003 & $79 \pm 9$ & 0.001 \\
\hline $\mathrm{UA}[\mu \mathrm{mo} / 1]$ & $308 \pm 80$ & $313 \pm 74$ & $313 \pm 102$ & 0.02 & $261 \pm 82$ & 0.001 \\
\hline Hcy $[\mu \mathrm{mo} / 1]$ & $12.6 \pm 6.4$ & $11.0 \pm 3.0$ & $12.2 \pm 5.3$ & 0.003 & $8.6 \pm 3.2$ & 0.001 \\
\hline Vit. B12 [pmol/1] & $283 \pm 125.4$ & $342 \pm 160.4$ & $332 \pm 110.7$ & 0.02 & $335 \pm 145.4$ & NS \\
\hline Folic acid [nmol/1] & $14.4 \pm 6.5$ & $15.1 \pm 9.7$ & $10.1 \pm 4.4$ & 0.001 & $12.8 \pm 8.6$ & NS \\
\hline $\mathrm{u}-\mathrm{CRP}[\mathrm{mg} / \mathrm{dl}]$ & $2.2 \pm 2.7$ & $2.6 \pm 2.9$ & $3.6 \pm 2.8$ & 0.002 & $3.1 \pm 3.0$ & NS \\
\hline
\end{tabular}

BMI - body mass index, SBP - systolic blood pressure, DBP - diastolic blood pressure, TCH - total cholesterol, LDL - LDL cholesterol, HDL - HDL cholesterol, TG triglycerides, ApoB - Apolipoprotein B, ApoA1 - Apolipoprotein A1, AI- atherogenic index, Hb - haemoglobin, Leu - leukocytes, Plt - platelets, $\mathrm{Fe}$ - iron, Bi - bilirubin, UA - uric acid, Hcy - homocystein, vit. B6 - vitamin B6, vit. B12 - vitamin B12, hsCRP - high sensitivity C-reactive protein, IHD - ischemic heart disease

Tab. 4. Prevalence of risk factors in males.

\begin{tabular}{|c|c|c|c|c|c|c|}
\hline \multicolumn{7}{|c|}{ Males } \\
\hline \multirow[t]{2}{*}{ Parameter } & \multicolumn{4}{|c|}{ IHD patients } & \multicolumn{2}{|l|}{ Controls } \\
\hline & Nové Zámky (N=60) & Bratislava $(\mathrm{N}=64)$ & Vel'ký Lom $(\mathrm{N}=31)$ & Sign. & Vel'ký Lom $(\mathrm{N}=47)$ & \\
\hline BMI $[\mathrm{kg} / \mathrm{m} 2] \geq 25$ & 90 & 81.3 & 74.2 & 0.02 & 72.3 & NS \\
\hline BMI $[\mathrm{kg} / \mathrm{m} 2] \geq 30$ & 40 & 33.3 & 38.7 & NS & 31.9 & NS \\
\hline $\mathrm{SBP}[\mathrm{mmHg}] \geq 140$ & 65 & 50 & 71 & NS & 51.1 & NS \\
\hline $\mathrm{DBP}[\mathrm{mmHg}] \geq 90$ & 50 & 33.5 & 71 & 0.001 & 64 & 0.02 \\
\hline $\mathrm{TCH}[\mathrm{mmol} / \mathrm{l}] \geq 5.0$ & 65.5 & 66.7 & 73.3 & NS & 85.1 & 0.001 \\
\hline LDL-C $[\mathrm{mmol} / \mathrm{l}] \geq 3.0$ & 69.1 & 75.8 & 76.7 & NS & 89.1 & 0.002 \\
\hline HDL-C $[\mathrm{mmol} / \mathrm{l}] \leq 1.1$ & 50 & 51.6 & 36.7 & 0.01 & 21.3 & 0.002 \\
\hline $\mathrm{TG}[\mathrm{mmol} / 1] \geq 1.7$ & 44.8 & 38.1 & 30.0 & NS & 29.8 & 0.058 \\
\hline Аро-B $[\mathrm{g} / \mathrm{l}] \geq 1.15$ & 34.5 & 22.8 & 30 & NS & 43.5 & 0.01 \\
\hline Apo-AI [g/l] $\leq 1.19$ & 93.1 & 96.5 & 80 & NS & 58.7 & 0.001 \\
\hline $\mathrm{AI} \geq 5.0$ & 46.7 & 43.2 & 50 & NS & 36.2 & 0.01 \\
\hline Smokers & 15 & 9.4 & 32.0 & & 46.8 & 0.001 \\
\hline AR $10 y \geq 20 \%$ & 88.3 & 74.6 & 66.6 & 0.05 & 25.5 & 0,001 \\
\hline Income $<$ average & 13.3 & 34.4 & 16.1 & 0.001 & 23.4 & NS \\
\hline Education & & & & & & \\
\hline Elementary & 15 & 7.8 & 38.7 & 0.001 & 31.9 & NS \\
\hline High school & 71.7 & 37.5 & 38.7 & 0.001 & 59.6 & NS \\
\hline University & 13.3 & 54.7 & 22.6 & 0.001 & 8.5 & NS \\
\hline Hcy $[\mu \mathrm{mo} / 1]>15$ & 30 & 37.5 & 32.3 & NS & 21.3 & NS \\
\hline Vitamin B6 [nmol/1] $\leq 0.6$ & 26.7 & 15.4 & 20.8 & NS & 25.7 & NS \\
\hline Vitamin B12 [pmol/1] $\leq 178$ & 18.3 & 15.3 & 26.7 & NS & 17.0 & NS \\
\hline Folic acid $[\mathrm{nmol} / 1] \leq 6.52$ & 30 & 32.2 & 66.7 & NS & 44.7 & NS \\
\hline $\mathrm{hsCRP}[\mathrm{mg} / \mathrm{dl}] \geq 0.3$ & 36.2 & 14 & 26.7 & 0.01 & 22.7 & NS \\
\hline
\end{tabular}

BMI - body mass index, SBP - systolic blood pressure, DBP - diastolic blood pressure, TCH - total cholesterol, LDL - LDL cholesterol, HDL - HDL cholesterol, TG triglycerides, ApoB - Apolipoprotein B, ApoA1 - Apolipoprotein A1, AI - atherogenic index, AR - absolute risk, Hcy - homocystein, hsCRP - high sensitivity C-reactive protein, IHD - ischemic heart disease 


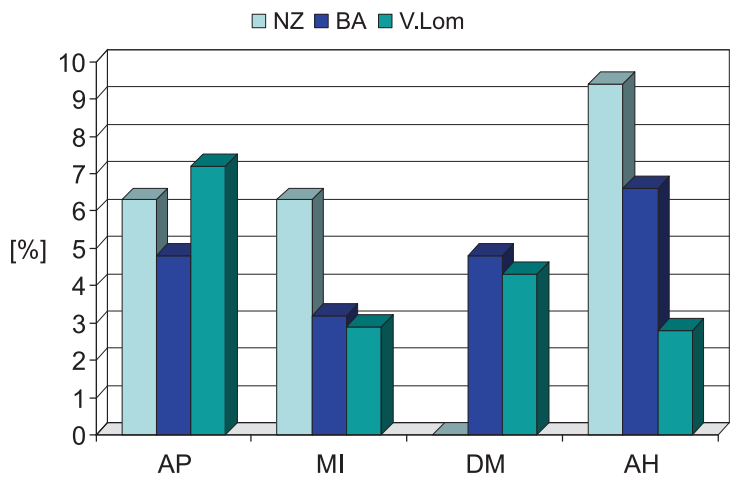

Fig. 1. Percentage of patients denying their diagnoses. AP - angina pectoris, MI - myocardial infarction, DM - diabetes mellitus, AH arterial hypertension.

in control women from Vel'ký Lom. The proportion of male IHD subjects with HCy level $>15 \mu \mathrm{mol} / 1$ was $30-38 \%$, and $21.3 \%$ for male controls from Vel'ký Lom (Tabs 2 and 3). The proportion of female IHD patients with HCy level $>13 \mu \mathrm{mol} / 1$ was highest in Nové Zámky, and $24.5 \%$ for Bratislava and Vel'ký Lom. Similarly to men, female controls from Vel'ký Lom had a lowest proportion of subjects with increased Hcy levels (Tabs 4 and 5). Suprisingly, both IHD and control subjects from the rural area in Vel'ký Lom had a tendency to yield lower serum levels of folic acid.

\section{Discussion}

Coronary heart disease (CHD) constitutes a major public health burden worldwide (17) and remains one of the most challenging topics in disease prevention. In spite of positive trends in decreasing cardiovascular mortality in Central European countries (CE) there remains a deep gap between European Union(EU) and Central European countries $(18,19)$. This gap cannot be explained solely by a difference in major risk factors. In fact, prevalence of certain classical risk factors in Western countries was higher than in former communist countries $(20,21,22)$ and it has been shown that over 10 years across the WHO MONICA Project populations, just $15 \%$ of coronary event rate trend variability in women and $40 \%$ in men could be explained by trends in major risk factors (21). The estimates are low, possibly due to imprecision in estimation of event rate trends; however, the data suggest that the remaining variance could be attributable to other factors. Mild hyperhomocysteinaemia was hypothesised to be an independent risk $(1,2)$ factor or marker $(6$, 23) for atherosclerosis and its complication. Nevertheless, the data on HCy levels distribution in Slovak population (24) are limited.

We therefore performed a study aimed at the description of prevalence of hyperhomocysteinaemia and major risk factors of atherosclerosis. We found a very high occurrence of risk factors regardless of geographic area (urban, urban/rural or rural) and health status of subjects (patients with IHD or general population). This finding is even more critical, when we consider that 6 out of 8 subgroups studied included patients in secondary prevention. The fact, that we have found a substantial part of patients to have an unfavourable profile of modifiable risk factors such as blood pressure, cholesterol levels, obesity and smoking suggests the limits of proper health care provided.

Our findings show the same trend with observations in previously published studies. In CINDI project in Central Slovakia,

Tab. 5. Prevalence of risk factors in females.

\begin{tabular}{|c|c|c|c|c|c|c|}
\hline \multicolumn{7}{|c|}{ Females } \\
\hline \multirow[t]{2}{*}{ Parameter } & \multicolumn{4}{|c|}{ IHD patients } & \multicolumn{2}{|l|}{ Controls } \\
\hline & Nové Zámky (N=70) & Bratislava $(\mathrm{N}=53)$ & Vel'ký Lom (N=53) & Sign. & Vel'ký Lom (N=55) & Sign. \\
\hline BMI $[\mathrm{kg} / \mathrm{m} 2] \geq 25$ & 92.9 & 75.5 & 90.6 & 0.02 & 65.5 & 0.001 \\
\hline BMI $[\mathrm{kg} / \mathrm{m} 2] \geq 30$ & 57.1 & 38.4 & 65 & NS & 43.6 & NS \\
\hline $\mathrm{SBP}[\mathrm{mmHg}] \geq 140$ & 82.9 & 71.2 & 83 & 0.02 & 45.5 & 0.001 \\
\hline $\mathrm{DBP}[\mathrm{mmHg}] \geq 90$ & 80 & 59.6 & 75.5 & 0.01 & 45.5 & 0.001 \\
\hline $\mathrm{TCH}[\mathrm{mmol} / \mathrm{l}] \geq 5.2$ & 89.4 & 84.0 & 96.2 & 0.03 & 88.9 & NS \\
\hline LDL-C $[\mathrm{mmol} / 1] \geq 3.5$ & 92.4 & 90.0 & 92.5 & 0.03 & 92.6 & NS \\
\hline HDL-C $[\mathrm{mmol} / 1] \leq 1.3$ & 43.9 & 41.5 & 26.4 & 0.001 & 25.9 & 0.02 \\
\hline $\mathrm{TG}[\mathrm{mmol} / \mathrm{l}] \geq 2.0$ & 39.1 & 26.0 & 30.2 & NS & 20.4 & 0.03 \\
\hline Apo-B $[\mathrm{g} / \mathrm{l}] \geq 1.15$ & 32.9 & 26.4 & 26.5 & NS & 37.8 & NS \\
\hline Apo-AI $[\mathrm{g} / \mathrm{l}] \leq 1.19$ & 60 & 75.5 & 36.5 & 0.001 & 51.9 & NS \\
\hline $\mathrm{AI} \geq 5.0$ & 35.7 & 37.3 & 57.7 & 0.02 & 29.6 & 0.03 \\
\hline Smokers & 4.3 & 3.8 & 7.5 & NS & 36.4 & 0.001 \\
\hline AR $10 y \geq 20 \%$ & 84.1 & 60.8 & 75.4 & NS & 15.4 & 0.001 \\
\hline Income $<$ average & 5.7 & 7.5 & 1.9 & NS & 9.1 & 0.002 \\
\hline Education & & & & & & \\
\hline Elementary & 50 & 28.3 & 65.4 & 0,002 & 32.7 & 0.01 \\
\hline High school & 45.7 & 56.6 & 30.8 & NS & 52.7 & NS \\
\hline University & 2.9 & 15.1 & 6.8 & NS & 9.1 & NS \\
\hline Hcy $[\mu \mathrm{mo} / 1]>13$ & 37.1 & 24.5 & 24.5 & NS & 7.3 & 0.001 \\
\hline Vitamin $\mathrm{B} 12 \leq 178$ & 14.3 & 15.1 & 1.9 & NS & 9.3 & NS \\
\hline Folic acid $\leq 6.52$ & 22.9 & 28.3 & 50 & NS & 35.2 & NS \\
\hline hsCRP $\geq 0.3$ & 30 & 34 & 50 & NS & 35.2 & NS \\
\hline
\end{tabular}

BMI - body mass index, SBP - systolic blood pressure, DBP - diastolic blood pressure, TCH - total cholesterol, LDL - LDL cholesterol, HDL - HDL cholesterol, TG triglycerides, ApoB - Apolipoprotein B, ApoA1 - Apolipoprotein A1, AI - atherogenic index, AR - absolute risk, Hcy - homocystein, hsCRP - high sensitivity C-reactive protein, IHD - ischemic heart disease 
more than 2,000 randomly selected respondents in age range of 15-64 were screened for major risk factors of atherosclerosis. In a subgroup of males in age range of 25-64 there was a prevalence of $26.5 \%$ in smokers, $66.2 \%$ with BMI $>25,66.3 \%$ with total cholesterol more than $5.2 \mathrm{mmol} / 1$, and $51.5 \%$ had blood pressure more than $140 \mathrm{and} /$ or $90 \mathrm{mmHg}$ (25). With a small difference in lower prevalence of smokers, the results of the latter study corresponded with our findings. Similarly, in a secondary prevention study VITA LONGA (with more than 4,000 participants with total cholesterol $>5.2$. $\mathrm{mmol} / \mathrm{l}$ or treated with hypolipidaemic treatment), the mean systolic and diastolic blood pressure and BMI were similar to those in our study. However, the proportion of smokers in VITA LONGA study was only $9.3 \%$ (26).

Both our study and other studies performed in Slovakia confirm high prevalence of classic and newer risk factors in our population. However, the lack of a systematic follow-up imposes a limit as to the predictive value of such information as well as to trends in risk factor changes over time. Therefore, it is important to perform a prospective study to obtain relevant data on such trends. This can form a base for a targeted population intervention focused on reversing the increasing trend of cardiovascular morbidity and mortality in Slovakia.

It has been suggested that low intake of vegetable and fruits can participate in the occurrence of mild hyperhomocysteinaemia in Slovakia $(24,27)$. However, there is only scarce data reporting plasma levels of HCY and modifying factors in Slovak population. In our study, we have found more than $30 \%$ of IHD male patients and $21.3 \%$ of males from control population with plasma Hcy levels over $15 \mu \mathrm{mo} / 1$. Similarly, in female IHD patients, the prevalence of Hcy levels $>13 \mu \mathrm{mo} / 1$ was in the range of $24.5-37.1$ $\%$ and $7.3 \%$ only in control females. In previously published study of Slovak men who survived myocardial infarction (MI) by the age of 50 and in control men without a personal and family history of MI, the mean plasma Hcy levels exceeded $15 \mu \mathrm{mo} / 1$ (24). These results suggest that mild hyperhomocysteinaemia can contribute to high cardiovascular morbidity and mortality in Slovakia. Surprisingly, rural patients who exhibited lower mean levels of Hcy in general had also lower serum levels of folic acid. This fact may suggest that folic acid level is not a crucial factor determining the plasma Hcy levels in our population.

In conclusion, we found a high prevalence of major risk factors of atherosclerosis and hyperhomocysteinaemia in selected Slovak population. This fact should be considered when developing national policies for IHD prevention.

Acknowledgement: The project has been sponsored by grants from the Grant Agency of Medical Faculty of Comenius University, of VEGA (Vedecká grantová agentúra Ministerstva školstva SR), of Grant Committee of Slovak Association of Atherosclerosis. Sponsorship has been provided also by TMC (Nitra) and technical and laboratory support by Roche company.

Especially we would like to thank Dr. Čipáková, prof. J.J.Genest Jr, prof. A. DeBold, prof. O. Hänninen, H. Pekkarinen, Ms. Paula Robble, Mr. Ján Bizov, Ing. Milan Backa, Ing. Milan Výboch, Mrs Eva Červeňová, Mrs. Anna Vyhňárová, Štefan Mikuš,
Dr. K. Gec, dr. Rakická, Dr Vachula, Prim. MUDr. O. Kollár, MUDr. Baltazár, MUDr. Dom. MUDr. Eliášová, MUDr. Gašpar, MUDr. Ilenčík, MUDr. Koval'číková, MUDr. Panenková, MUDr. Sedláčková, MUDr. Surkošová, Prim. MUDr. Stuhlrajter, MUDr. Škreko, MUDr. Viera Strolková, MUDr. Jaromír Likér, MUDr. Marian Karvay, Dr. Ivan Rovný, Dr. Krkoška, Dr. Beňačka, Mrs J. Kotrecová, Ms. V. Némethová, Doc. MUDr. J. Trupl, MUDr. J. Lakota, Dr. Urbančíková, Dr. M. Dušínska, Mr. Valach, MUDr. M. Kotrec, MUDr. V. Krpčiar, MUDr. P. Kuka, MUDr. R. Surovčík, MUDr. P. Vážny, MUDr. J. Zimanová, MUDr. M. Belošovičová, L. Sarkozyová, J. Cziszárová, M. Svitková.

L. Bartaloš, M. Čaprnda, B. Duchaj, M. Hanuska, J. Heteni, V. Husárová, K. Jarošová, J. Kralová, D. Križanová, P. Penc, M. Príhodová, M. Rakovan, J. Štrelinger, J. Taliga, A. Teren, I. Vacula, A. Žemberová, K. Žilinčanová.

\section{References}

1. Wald DS, Law M, Morris JK. Homocysteine and cardiovascular disease: evidence on causality from a meta-analysis. BMJ 2002; 325: 1202-1207.

2. Homocysteine Studies Collaboration. Homocysteine and risk of ischemic heart disease and stroke: a meta-analysis. JAMA 2002; 288 : 2015-2022.

3. Boushey CJ, Beresford SAA, Omenn GS et al. A quantitative assessment of plasma homocysteine as a risk factor for vascular disease. Probable benefits of increasing folic acid intakes. JAMA 1995; 274: 1049-1057.

4. Brattström L, Wilcken DEL. Homocysteine and cardiovascular disease: cause or effect? Am J Clin Nutr 2000; 72: 315-323.

5. Ueland PM, Refsum H, Beresford SAA, Vollset SE. The controversy over homocysteine and cardiovascular risk. Am J Clin Nutr 2000; 72: 324-332

6. Lonn E, Yusuf S, Arnold MJ, Sheridan P, Pogue J, Micks M, McQueen MJ, Probstfield J, Fodor G, Held C, Genest J Jr; Heart Outcomes Prevention Evaluation (HOPE) 2 Investigators. Homocysteine lowering with folic acid and B vitamins in vascular disease. N Engl J Med 2006; 354 (15): 1567-1577.

7. Bonaa KH, Njolstad I, Ueland PM, Schirmer H, Tverdal A, Steigen T, Wang H, Nordrehaug JE, Arnesen E, Rasmussen K; NORVIT Trial Investigators. Homocysteine lowering and cardiovascular events after acute myocardial infarction. N Engl J Med 2006; 354 (15): 1578-1588.

8. Zdravotnícka ročenka Slovenskej republiky 2007. Bratislava: Národné centrum zdravotníckych informácií, 2008, 195 pp.

9. Baráková A (Ed). Vybrané informácie zo zdravotníckej štatistiky o vývoji ochorení obehovej sústavy v SR. Projekt MONIKA. Bratislava: Štátny zdravotný ústav SR 1999: 111.

10. Geochemical atlas of Slovakia, Part IV: Natural Rock Activity, Bratislava: Štátny Geologický ústav SR 1999: 99.

11. International Labour Office. Bureau of Statistics. International standard classification of. occupations (ISCO 88) Geneva, International Labour Office, 1991: 457.

12. Rose GA., Blackburn H., Gillum FR, Prineas RJ. Cardiovascular survay methods, 2nd ed, Geneva: World Health Organisation 1982 Monograph Serial No 56: 123-129. 
13. Task Force Committee. Prevention of coronary heart disease in clinical practice. Recommendations of the Second Joint Task Force of European and other Societies on Coronary prevention. Eur Heart J 1998; 19: $1434-1503$

14. Novák M. O kouření. Praha: Avicenum, 1975: 154.

15. Friedewald WT, Levy RI, Fredrickson DS. Estimation of the concentration of the low-density lipoprotein cholesterol in plasma without the use of preparative ultra centrifugation. Clin Chem 1972; 18: 499-509.

16. Atalay M, Sen CK. Physical exercise and antioxidant defence in the heart. Ann NY Acad Sci 1999; 874: 169-167.

17. Murray CJL, Lopez AD. Mortality by cause for eight regions of the world: Global Burden of Disease Study. Lancet 1997; 349: 1269-1276.

18. Ginter E. Health-status of the Slovak Republic population in comparison with countries of Central Europe and European Union. Bratisl Lek Listy 2000; 101: 589-593.

19. Müller-Nordhorn J, Binting S, Roll S, Willich SN. An update on regional variation in cardiovascular mortality within Europe. Eur Heart J. 2008; 29: 1316-1326.

20. Ginter E. High Cardiovascular Mortality in Postcommunistic Countries: Participation of Oxidative Stress? Internat J Vit Nutr Res 1996; 66: $183-189$
21. Kuulasmaa K, Tunstall-Pedoe H, Dobson A, et al. Estimation of contribution of changes in classic risk factors to trends in coronary-event rates across the WHO MONICA Project populations. Lancet 2000; 355: $675-687$.

22. Dukát A, Lietava J, Krahulec B, Caprnda M, Vacula I, Sirotiaková J, Kosmálová V, Minárik P; IDEA Slovakia. [The prevalence of abdominal obesity in Slovakia. The IDEA Slovakia study] 2007; 53 (4): 326-330.

23. Milani RV, Lavie CJ. Homocysteine: The Rubik's Cube of Cardiovascular Risk Factors. Mayo Clin Proc. November 2008; 83 (11): 1200-1202.

24. Rašlová K, Smolková B, Vohnout B et al. Risk factors for atherosclerosis in survivors of myocardial infarction and their spouses: comparison to controls without personal and family history of atherosclerosis. Metabolism 2001; 50: 24-29.

25. Baráková A, a kol. Vybrané informácie zo zdravotníckej štatistiky o vývoji ochorení obehovej sústavy v SR. Bratislava: ŠZÚ SR, 1999: 95-109.

26. Kamenský G, Šidlo R, Mikeš Z et al. VITA LONGA, program sekundárnej prevencie koronárnej choroby srdca pacientov s hypercholesterolémiou: výsledky dvojročného sledovania. Cardiol 2003; 12: 116-128.

27. Schifferdecker B, Vohnout B. Hyperhomocyst(e)inémia, rizikový faktor kardiovaskulárnych chorôb a jej liečba. Cardiol 2000; 9: 66-74.

Received December 7, 2010. Accepted December 18, 2011. 